\title{
VARIABILIDADE ESPACIAL DA POROSIDADE DRENÁVEL DE UM SOLO DE VÁRZEA, NO MUNICÍPIO DE PIRACICABA, ESTADO DE SÃO PAULO
}

\author{
José Elenildo Queiroz ${ }^{1}$, Décio Eugênio Cruciani ${ }^{2}$ \& Paulo Leonel Libardi ${ }^{3}$
}

\begin{abstract}
RESUMO
Este trabalho foi desenvolvido com o objetivo de se estudar a variabilidade espacial da porosidade drenável de um solo de várzea (Gleissolo Eutrófico, textura argilosa) empregando-se técnicas estatísticas descritivas e geoestatísticas na análise dos dados. Para tanto, numa área de 10 x 90 m definiu-se uma malha com espaçamento regular de 5,00 m entre linhas e 3,33 m entre colunas, da qual foram extraídas 84 amostras de solo com estrutura indeformada (profundidade média de $0,30 \mathrm{~m}$ ) para determinação da porosidade drenável, usando-se uma mesa de tensão. As análises estatísticas mostraram que os dados se ajustaram a uma distribuição normal e apresentaram média variabilidade (CV $=43,8 \%)$ além de uma fraca dependência espacial entre as observações (efeito pepita $>75 \%$ ) com alcance médio de $23 \mathrm{~m}$. Ao semivariograma experimental obtido foi ajustado um modelo esférico, cujos parâmetros foram empregados no processo de krigagem, o que permitiu a elaboração de um mapa de isovalores de porosidade drenável, cuja interpretação é de grande importância no planejamento do sistema de drenagem.
\end{abstract}

Palavras-chave: drenagem subterrânea, porosidade drenável, variabilidade espacial

\section{SPATIAL VARIABILITY OF DRAINABLE POROSITY OF A HOLM SOIL IN PIRACICABA, SÃO PAULO STATE}

\begin{abstract}
This research was developed with the purpose to study the spatial variability of the drainable porosity of a holm soil, using descriptive statistical techniques and geostatistics in the analysis of the data. Undisturbed soil samples were collected (average depth of $0.30 \mathrm{~m}$ ) in a grid of 84 points, with regular spacing of $5.00 \mathrm{~m}$ between rows and $3.33 \mathrm{~m}$ between columns, within a field plot of $10 \times 90 \mathrm{~m}$, for determination of the drainable porosity using a tension table. The statistical analyses showed that the data were adjusted to a normal distribution and presented average variability $(\mathrm{CV}=43.8 \%)$, as well as weak spatial dependence between the observations (nugget effect $>75 \%$ ), with range medium of $23 \mathrm{~m}$. To the obtained experimental semivariogram a spherical model was adjusted, whose parameters were used in the kriging process, which allowed the elaboration of a map of isovalues of drainable porosity, whose interpretation is important in the planning of the underground drainage system.
\end{abstract}

Key words: underground drainage, drainable porosity, spatial variability

\footnotetext{
${ }^{1}$ Prof. Adjunto Dept. de Engenharia Florestal/UFPB/Campus VII, CP 64, CEP 58700 - 970, Patos, PB. E-mail: elenildo@ cstr.ufpb.br

${ }^{2}$ Prof. Titular Dept. Engenharia Rural da ESALQ/USP, CEP 13418 - 900, Piracicaba, SP. Fone: (019) 429 4217, Fax: (019) 433 0934

${ }^{3}$ Prof. Titular Dept. de Física e Meteorologia da ESALQ/USP, CEP 13418 - 900, Piracicaba, SP. Fone: (019) 422 5925, Fax: (019) 434 5259
} 


\section{INTRODUÇÃO}

A variabilidade espacial do solo, o excesso de umidade e flutuações do lençol freático, entre outros fatores, constituem problemas que dificultam a incorporação dos terrenos de várzeas ao processo produtivo (Goedert, 1985). Em decorrência de fatores inerentes a cada local, processos de formação e desenvolvimento do solo e das práticas de manejo utilizadas, uma ampla variabilidade espacial pode ocorrer nos valores de porosidade drenável.

Nas análises estatísticas usuais supõe-se que os valores obtidos de determinada propriedade ou característica do solo sejam aleatórios e espacialmente independentes, o que nem sempre é verdade. Machado (1994) por exemplo, ao estudar a variabilidade espacial de uma hidrosseqüência de solos bem a mal drenados, encontrou uma ampla variação nos resultados de porosidade drenável ou macroporosidade tendo, através de análises geoestatísticas, encontrado dependência espacial entre as observações, para a maioria das situações estudadas.

A utilização de ferramentas geoestatísticas, além de identificar a existência ou não de dependência espacial entre as observações, pode ser aplicada em mapeamento, orientação de futuras amostragens e modelagem. Se os valores próximos não são independentes entre si, pode-se usar ferramentas mais sofisticadas de interpolação e estimação de valores em lugares não medidos, a exemplo do processo de krigagem; desta forma, uma representação mais realista da área estudada pode ser obtida (Isaaks \& Srivastava, 1989; Vieira, 1995).

Uma análise adequada dos dados de porosidade drenável constitui aspecto de fundamental importância no planejamento do sistema de drenagem subterrânea. A compreensão da estrutura de dependência espacial entre as observações permite separar a região estudada em sub-regiões de menor variabilidade, tornando possível o dimensionamento do sistema de drenagem em função de valores representativos de cada subregião.

Este trabalho teve como objetivo estudar a variabilidade da porosidade drenável de um solo de várzea (Gleissolo eutrófico, textura argilosa) empregando-se técnicas estatísticas descritivas e procedimentos geoestatísticos na análise dos dados, visando fornecer subsídios que permitam orientar melhor o planejamento de experimentos envolvendo estudos de drenagem subterrânea na área estudada.

\section{MATERIAL E MÉTODOS}

A área onde os trabalhos experimentais foram desenvolvidos localiza-se no Campo Experimental de drenagem do Departamento de Engenharia Rural, Campus da Escola Superior de Agricultura “Luiz de Queiroz", Universidade de São Paulo, município de Piracicaba, Brasil, tendo como coordenadas geográficas: $22^{\circ} 42^{\prime} 30^{\prime \prime}$ de latitude sul, $47^{\circ} 38^{\prime} 00^{\prime \prime}$ de longitude oeste e altitude média de $546 \mathrm{~m}$. O solo é classificado como um Gleissolo eutrófico, horizonte A moderado, textura argilosa a média (Typic Haplaquet) com inclusões de solos vérticos (Vertic Haplaquet) conforme Vidal-Torrado \& Sparovek (1993).

O esquema experimental foi definido de modo a permitir uma amostragem sistemática numa área de 10 × 90 m, onde se definiu uma malha com espaçamento regular de 5,00 m entre linhas e $3,33 \mathrm{~m}$ entre colunas, totalizando 84 pontos. De cada ponto extraiu-se uma amostra de solo com estrutura indeformada, na profundidade média de $0,30 \mathrm{~m}$, mediante a utilização de anéis volumétricos de bordas cortantes de $0,03 \mathrm{~m}$ de altura e $0,047 \mathrm{~m}$ de diâmetro, aproximadamente. As determinações foram realizadas no Laboratório de Física de Solos do Departamento de Engenharia Rural da ESALQ/USP. As amostras, depois de saturadas, foram colocadas numa mesa de tensão e submetidas a sucção de $6 \mathrm{kPa}$, até atingirem a condição de equilíbrio (Learmer \& Shaw, 1941; Oliveira, 1968). Após esta condição, as amostras foram retiradas da mesa de tensão e pesadas novamente. Com o volume das amostras e os pesos correspondentes à saturação e sucção de $6 \mathrm{kPa}$, calculou-se a porosidade drenável (Coelho, 1984).

As análises estatísticas foram realizadas através de técnicas descritivas e geoestatísticas. O objetivo das análises descritivas foi observar o comportamento geral dos dados, enquanto as geoestatísticas tiveram, como objetivo, identificar a estrutura de variabilidade dos dados. As análises descritivas consistiram no cálculo de medidas estatísticas, na distribuição de frequiências e na análise do comportamento espacial dos dados, através de gráficos de dispersão por linhas e colunas. Nas análises geoestatísticas, a fim de identificar a estrutura de dependência espacial entre as observações, utilizou-se a função semivariância, expressa pela seguinte equação (Vieira et al., 1983; Dourado Neto, 1989; Queiroz, 1995):

$$
\hat{\gamma}(h)=\frac{1}{2 N(h)} \sum_{i=1}^{N(h)}\left[Z\left(x_{i}\right)-Z\left(x_{i}+h\right)\right]^{2}
$$

em que:

$\hat{\gamma}($ h) é a estimativa da semivariância, $\mathrm{N}(\mathrm{h})$ o número de pares de observações e h é o vetor que separa os pares de observações Z(xi)e Z(xi+h).

A partir dos valores de semivariância construiu-se o semivariograma experimental para o conjunto de dados, assumindo-se a hipótese de isotropia, isto é, mesmo comportamento da porosidade drenável nas diferentes direções do espaço. Ao semivariograma experimental ajustou-se um modelo teórico, cujos parâmetros foram utilizados na interpolação de valores em pontos não amostrados pela técnica de krigagem. Para avaliação do melhor modelo e do melhor número de vizinhos, utilizou-se a técnica conhecida como "jack-knifing" (Vieira et al., 1983).

A partir de um total de 275 pontos, estimados numa área retangular de 10 x $90 \mathrm{~m}$, numa malha com linhas separadas por 2,50 $\mathrm{m}$ e colunas por $1,65 \mathrm{~m}$, construiu-se um mapa de isolinhas de porosidade drenável, a partir do qual se pode definir zonas de menor variabilidade e obter valores representativos para o dimensionamento do sistema de drenagem subterrânea por sub-regiões.

\section{RESULTADOS E DISCUSSÃO}

As medidas estatísticas descritivas dos dados de porosidade drenável, expressos em porcentagem, são apresentadas na Tabela 1, na qual se observa variação de 2,17 a 14,31\% (6,6 vezes) com média $6,41 \%$, mediana $6,40 \%$, desvio-padrão de 
$2,81 \%$ e amplitude total $12,14 \%$. Esses resultados estão dentro dos limites normalmente encontrados em solos argilosos e argiloarenosos (Pizarro, 1978). O valor da mediana, aproximadamente igual à média aritmética, é um indicativo de possível normalidade dos dados e o coeficiente de variação de $43,83 \%$ indica média magnitude de variabilidade dos dados. Uma variação dessa ordem de grandeza, em um solo de várzea tipo orgânico $(\mathrm{HO})$ também foi encontrada por Machado (1994).

Tabela 1. Resumo estatístico dos dados de porosidade drenável $(\mu)$ obtidos a partir de 84 determinações

\begin{tabular}{lr}
\hline Estatísticas & $\mu(\%)$ \\
\hline Média & 6,41 \\
Mediana & 6,40 \\
Variância & 7,90 \\
Desvio-padrão & 2,81 \\
CV (\%) & 43,83 \\
Mínimo & 2,17 \\
Máximo & 14,31 \\
Amplitude total (At) & 12,14 \\
Quartil inferior & 4,10 \\
Quartil superior & 8,09 \\
Amp. interqualítica (Ai) & 3,99 \\
Assimetria & 0,59 \\
Curtose & 3,08 \\
\hline
\end{tabular}

A análise dos quartis mostra que 50\% dos dados de porosidade drenável, situados entre o quartil inferior e o quartil superior, pertencem ao intervalo de 4,10 a 8,09, o que corresponde a uma amplitude interquartílica igual a 3,99. Esta variação corresponde a apenas $33 \%$ da dispersão total dos dados, indicando que a amplitude total representa uma medida de dispersão influenciada por valores extremos, possivelmente "atípicos".

Os coeficientes de assimetria e curtose, para um nível de significância de 5\%, indicam um comportamento aproximadamente normal dos dados (Jones, 1969). O teste de aderência de Kolmogorov-Smirnov (K-S) aplicado a nível de significância de 5\%, reforça a aceitação da hipótese de normalidade dos dados, isto é, os valores de porosidade drenável podem ser considerados provenientes de uma população com distribuição normal de média 6,41 e variância 7,90. Para esse nível de significância, o intervalo de confiança apresenta limite inferior igual a 5,81 e limite superior igual a 7,01.

Embora o conjunto dos dados de porosidade drenável se tenha ajustado a uma distribuição normal, o máximo valor obtido $(14,31 \%)$ é maior que o limite superior de dados não discrepantes, isto é, maior que o quartil superior, adicionado 1,5 vezes à dispersão dos quartis. O limite inferior para dados discrepantes ou "outliers" (quartil inferior menos 1,5 vezes a dispersão dos quartis) corresponde ao valor $-1,88$, o qual, por ser negativo, não tem significado físico para porosidade drenável. A análise descritiva sem o valor discrepante $(14,31 \%)$ mostrou que as principais medidas estatísticas não foram afetadas, podendo-se afirmar que se trata de um dado não influente, mas nas análises geoestatísticas é recomendável se calcular as semivariâncias com e sem este dado, a fim de se verificar se ele provoca algum problema na obtenção do semivariograma experimental.
À luz da estatística clássica, em que as observações são consideradas aleatórias e independentes espacialmente, como os dados de porosidade drenável se ajustaram à distribuição normal, a média aritmética $(6,41 \%)$ pode ser considerada uma medida de tendência central representativa do conjunto de dados, podendo ser utilizada no dimensionamento do sistema de drenagem subterrânea. Todavia, com este procedimento o sistema poderá ser superdimensionado em alguns setores e subdimensionado em outros.

O comportamento dos dados em relação às direções da malha experimental é mostrado na Figura 1. Observa-se, na Figura 1A, que os dados apresentam comportamento semelhante nas três linhas, embora seja observada uma dispersão ligeiramente menor na terceira linha (distância $\mathrm{Y}=12,5 \mathrm{~m}$ ). A dispersão dos dados por coluna, representados na Figura 1B, mostra que as variações, com algumas exceções, são muito semelhantes. Estas análises indicam não haver tendência claramente observável nas direções da malha experimental, o que significa não existir, também, maiores problemas ao assumir a hipótese de estacionaridade dos dados.

\section{A. Dispersão por linha}
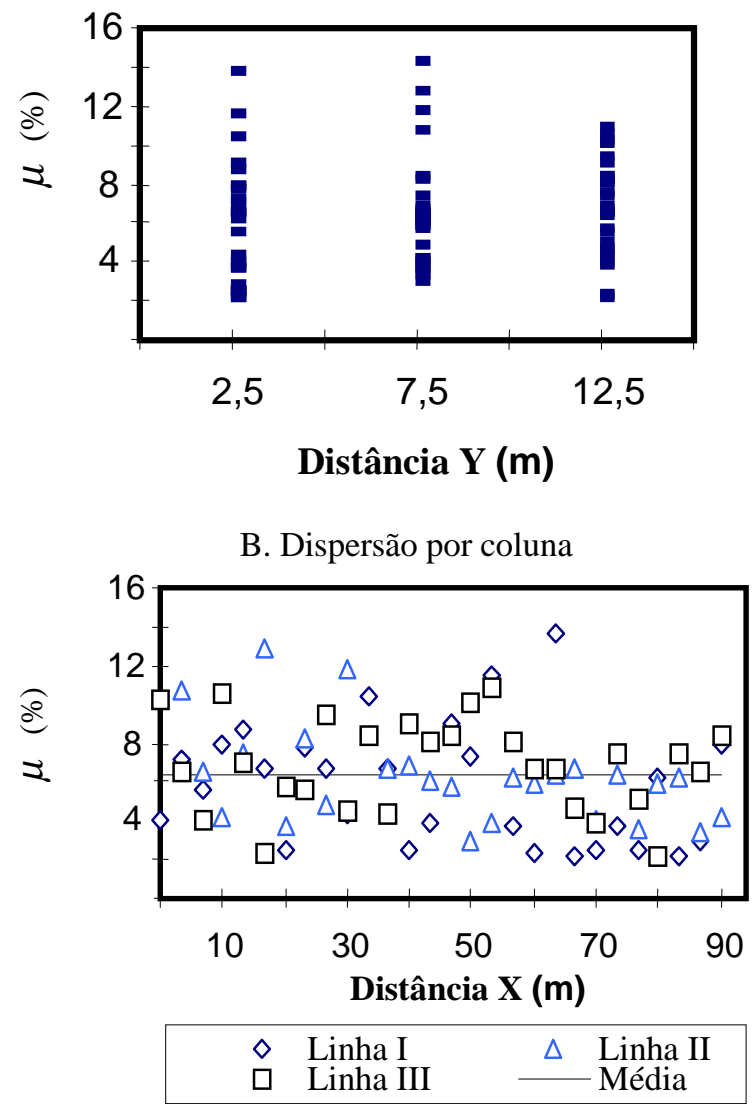

Figura 1. Gráficos de dispersão dos dados de porosidade drenável $(\mu)$ por linha (A) e coluna (B) expressos em percentagem

A partir das indicações da análise descritiva, a fim de se verificar o efeito do dado discrepante, utilizando-se o programa computacional desenvolvido por Vieira et al. (1983) várias tentativas e avaliações foram realizadas para estimativa dos semivariogramas experimentais, com e sem eliminação do dado considerado "atípico". Depois da avaliação de vários 
semivariogramas, decidiu-se considerar o semivariograma experimental obtido com eliminação do valor discrepante $(14,31 \%)$ o qual teve influência nos valores de semivariâncias. Após análise comparativa entre vários modelos de semivariograma, o modelo esférico apresentou melhor ajuste, sendo a melhor opção para um número de 16 vizinhos. Os parâmetros do modelo foram: "nugget effect" $(\mathrm{Co})=5,3$; patamar $(\mathrm{Co}+\mathrm{C} 1)=6,8$ e alcance de dependência espacial (a) $=23 \mathrm{~m}$. O "nugget effect" (efeito pepita), expresso como uma percentagem do patamar (valor igual a 78\%), indica elevada influência da componente aleatória na variabilidade espacial dos dados. De acordo com os critérios propostos por Cambardella et al. (1994) o efeito pepita igual a 78\% demonstra uma fraca dependência espacial da variável avaliada.

Com os parâmetros do modelo esférico ajustado ao semivariograma, obtém-se a seguinte expressão:

$$
\begin{gathered}
\hat{\gamma}(h)=5,3+1,5\left[\frac{3}{2}\left(\frac{h}{23}\right)-\frac{1}{2}\left(\frac{h}{23}\right)^{3}\right] \quad 0<h \leq 23 \\
\hat{\gamma}(h)=6,8 \quad h>23
\end{gathered}
$$

O semivariograma experimental com o respectivo modelo ajustado pode ser visualizado na Figura 2. O alcance de $23 \mathrm{~m}$ indica a amplitude de correlação espacial entre as observações, representando a distância em que a utilização das técnicas geoestatísticas conduz a estimativas com maior precisão. Esta relação de dependência espacial implica em interação entre os dados, os quais não podem ser considerados independentes dentro do domínio de $23 \mathrm{~m}$.

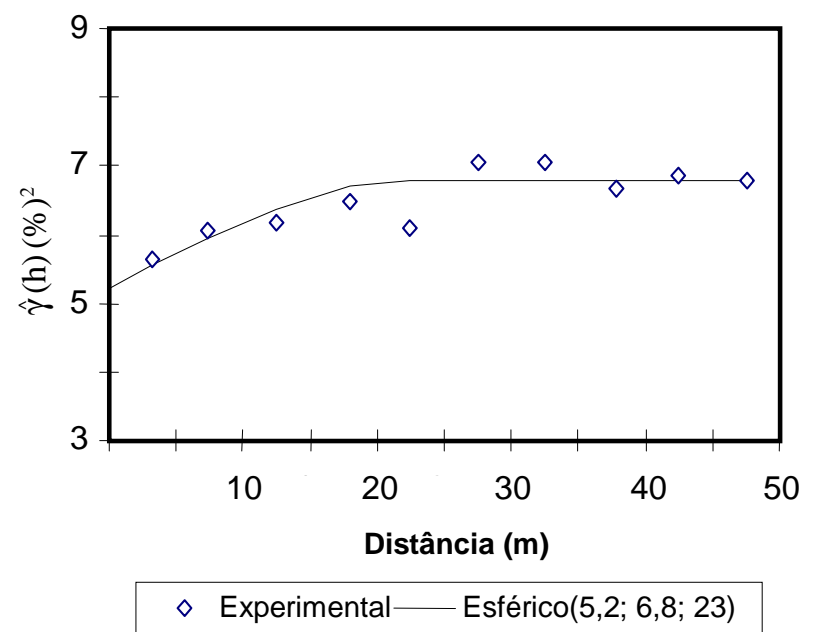

Figura 2. Semivariograma experimental mostrando o modelo esférico ajustado para os dados de porosidade drenável

Com os parâmetros do modelo ajustado ao semivariograma e o número de vizinhos, definidos pela técnica do "jack-knifing", utilizando-se o processo de interpolação por krigagem, foram estimados valores de porosidade drenável em uma área retangular de $10 \times 90 \mathrm{~m}$, numa malha com linhas separadas por $1,65 \mathrm{~m}$ e colunas por $2,5 \mathrm{~m}$, totalizando 275 pontos. A partir dos valores estimados, construiu-se o mapa de isolinhas apresentado na Figura 3. As pequenas áreas, com a ocorrência de muitas linhas de contorno fechadas, indicam flutuações nos valores de porosidade drenável, isto é, valores altos e baixos a pequenas distâncias. Através do mapa de isolinhas pode-se fazer um planejamento melhor do sistema de drenagem subterrânea, levando-se em conta a variabilidade espacial dos parâmetros envolvidos no dimensionamento do sistema; com isto, o dimensionamento do sistema pode ser feito a partir de valores representativos de cada sub-região, definidas como zonas de menor variabilidade como, por exemplo, as sub-regiões I e II, indicadas na Figura 3.

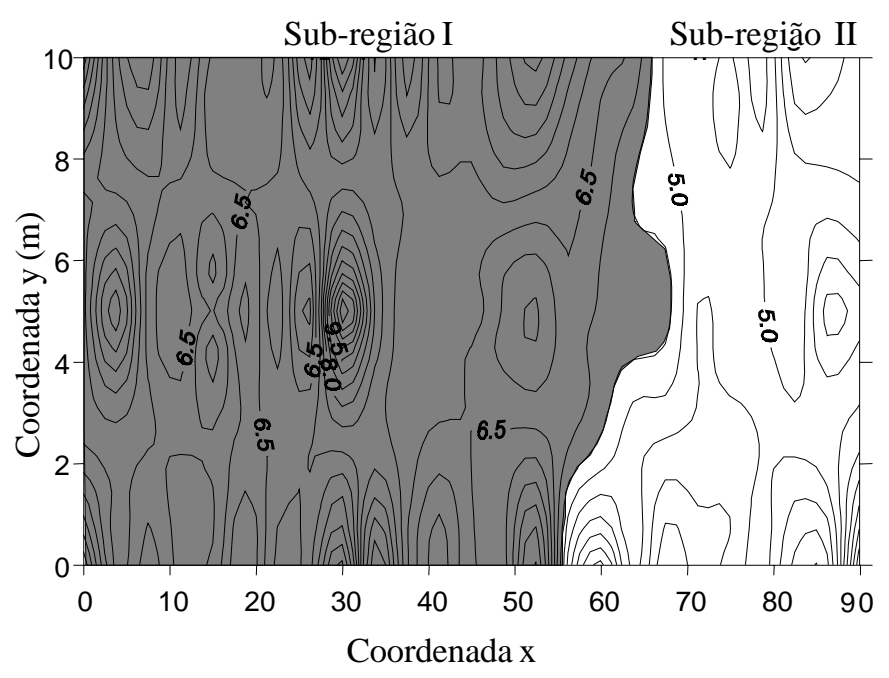

Figura 3. Mapa de isolinhas de porosidade drenável, expressa em porcentagem

\section{CONCLUSÕES}

Com base na análise dos resultados obtidos neste trabalho, pode-se concluir que:

1. A porosidade drenável apresentou distribuição normal, variabilidade média $(\mathrm{CV}=43,83 \%)$ e fraca estrutura de dependência espacial, com alcance médio da ordem de $23 \mathrm{~m}$.

2. A dependência espacial encontrada mostra que a porosidade drenável, embora tenha apresentado grande aleatoriedade, caracterizada pelo alto "nugget effect", não pode ser tratada como completamente aleatória, indicando que no planejamento de novas amostragens, o alcance da dependência espacial deve ser considerado.

3. O mapeamento da área pelo processo de krigagem permitiu a definição de zonas de menor variabilidade para fins de planejamento e estudos envolvendo drenagem subterrânea na área estudada.

\section{REFERÊNCIAS BIBLIOGRÁFICAS}

CAMBARDELLA, C.A.; MOORMAN, T.B.; NOVAK, J.M.; PARKIN, T.B.; KERLEN, D.L.; TURCO, R.F.; KONOPKA, A.E. Field scale variability of soil properties in Central Iowa soils. Soil Science Society America Journal, Madison, v.47, p.1501-1511, 1994.

COELHO, E.F. Desempenho de algumas equações de drenagem e dos drenos abertos e cobertos em condições de campo. Viçosa: UFV, 1984. 80p. Dissertação Mestrado 
DOURADO NETO, D. Variabilidade espacial das alturas de chuva e irrigação e de potenciais da solução do solo. Piracicaba: ESALQ/USP, 1989. 180p. Dissertação Mestrado

GOEDERT, W.J. Solos dos cerrados: tecnologias e estratégias de manejo. São Paulo: Nobel; Brasília: EMBRAPA - CPAC, $1985.422 \mathrm{p}$.

ISAAKS, H.E.; SRIVASTAVA, R.M. An introduction to applied geostatistes. New York: Oxford University Press, 1989. 560p.

JONES, T.A. Skewness and kurtosis as criteria of normality in observed frequency distributions. Journal Sedimentary Petrology, Houston, p.1622-1627, Dec. 1969.

LEARMER, R.W.; SHAW, B. A simple apparatus for measuring non capillary porosity on an extensive scale. Journal of American Society of Agronomy, Madison, v. 33, p.1003-1008, 1941.

MACHADO, R.V. Variabilidade espacial de atributos físicohídricos em uma hidrossequência de solos bem a muito mal drenados. Lavras: Escola Superior de Agricultura de Lavras, 1994. 88p. Dissertação Mestrado
OLIVEIRA, L.B. Determinação da macro e microporosidade pela mesa de tensão em amostras de solo com estrutura indeformada. Pesquisa Agropecuária Brasileira, Brasília, v.3, p.197-200, 1968.

PIZARRO, F. Drenaje agrícola y recuperación de suelos salinos. Madrid: Agrícola Espanhola, 1978. 525p.

QUEIROZ, J.E.Parâmetros hidrodinâmicos de um solo de várzea para fins de drenagem subterrânea. Piracicaba: ESALQ/USP, 1995. 167p. Tese Doutorado

VIDAL-TORRADO, P.; SPAROVEK, G. Mapa pedológico detalhado do Campus "Luiz de Queiroz". Piracicaba: ESALQ, 1993. Escala 1:10.000

VIEIRA, S.R. Curso de atualização em geoestatística. Campinas: Instituto Agronômico, 1995. p.1, 76p.

VIEIRA, S.R.; HATFIELD, J.L.; NIELSEN, D.R.; BIGGAR, J.W. Geostatistical theory and application to variability of some agronomical properties. Hilgardia, Califórnia, v.31, n.3, 1983, $75 \mathrm{p}$. 